\title{
HARMONIC CAVITIES AND LONGITUDINAL BEAM STABILITY IN ELECTRON STORAGE RINGS*
}

\author{
J. M. Byrd ${ }^{\dagger}$, S. De Santis, G. Stover, LBNL, Berkeley, CA 85710 USA \\ Dmitry Teytelman, John Fox, SLAC, Stanford, CA \\ J. Jacob, V. Serriere, ESRF, Grenoble, France \\ M. Georggson, MAXLab, Lund, Sweden
}

\begin{abstract}
Harmonic cavities have been used in storage rings to increase beam lifetime and Landau damping by lengthening the bunch. The need for lifetime increase is particularly great in the present generation of low to medium energy synchrotron light sources where the small transverse beam sizes lead to relatively short lifetimes from large--angle intrabeam (Touschek) scattering. We review the beam dynamics of harmonic radiofrequency (RF) systems and discuss effects on longitudinal beam stability.
\end{abstract}

\section{INTRODUCTION}

Harmonic cavities have enjoyed a resurgence of interest in the past few years in the present generation of lowmedium energy synchrotron light sources due to the severe effect on the beam lifetime from Touschek scattering. The main function of the harmonic cavities in a synchrotron light source is to generate a voltage which counteracts the longitudinal focussing at the bunch center of the main RF voltage, lengthening the bunch, decreasing the charge density and thus improving the Touschek-dominated lifetime.

In addition to the basic effect described above, the reduction of longitudinal focussing can have a number of other desirable and undesirable effects on the longitudinal stability of the ring, particularly in the presence of multibunch instabilities and feedback systems. During the recent commissioning of the harmonic RF system at the Advanced Light Source, we experienced many of these effects. This paper summarizes our experiences and, wherever possible, provides an explanation along with a model of the effect which can be applied to the study of harmonic systems at other rings. Much of the details can be found elsewhere[1-4]. Reports on other harmonic systems[5-12] and longitudinal dynamics can found elsewhere[13-16]

An introduction to harmonic RF with emphasis on passive cavities is given in Section 2. Our experience at the ALS is summarized in section 3. The issue of beam loading transient effects has been found to be important for asymmetric beam fill patterns and is discussed in Section 4. A summary and conclusions are given in Section 5.

\footnotetext{
* Work supported by the U.S. Department of Energy under Contract Nos. DE-AC03-76SF00098..

$\dagger$ JMByrd@lbl.gov
}

\section{INTRODUCTION TO HARMONIC CAVITIES}

Consider the voltage seen by the bunch generated by the main RF system as shown in Fig. 1. Near the bunch center, the restoring force of the RF voltage is approximately linear. Given a gaussian energy spread, the resulting longitudinal distribution is also gaussian. If another voltage is added to the main RF voltage with an amplitude and phase such that the slope at the bunch center is zero, the energy distribution is unaffected but the bunch lengthens and the peak charge density decreases and the lifetime improves. To achieve cancellation on every RF cycle, the frequency of the secondary voltage must be a higher harmonic of the main RF voltage.

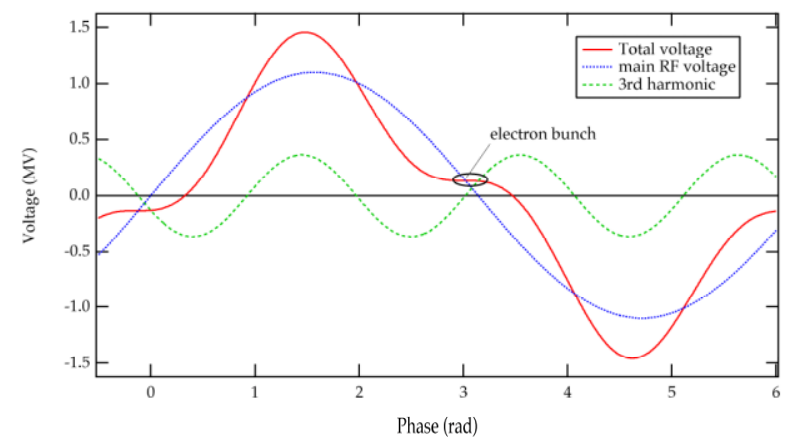

Figure 1. Main and harmonic voltages adjusted to cancel the longitudinal focussing at the bunch center.

A higher harmonic cavity has several other benefits to machine operation. When the phase of the harmonic voltage is adjusted such that the bunch lengthens, there is an increase in the spread of synchrotron frequencies within the bunch. This spread can help in damping coherent instabilities such as the longitudinal coupled bunch instabilities through Landau damping. For this reason, harmonic cavities are often called "Landau" cavities.

To produce the harmonic voltage, a second RF cavity system is installed in the ring with a resonant frequency several times the main RF cavity. The voltage in the harmonic cavity is generated either by an external generator (i.e. active cavity) or by the beam itself (passive cavity). Typically the harmonic RF system is introduced in passive mode and subsequently upgraded to active mode. One of the limitations of operating in passive mode is that the optimum bunch lengthening conditions can only be reached at one value of the beam current. 
The potential well and bunch distribution for the main RF voltage and with the harmonic voltage adjusted to perfectly cancel the main RF voltage are shown in Figure 2a. Note that these conditions do not necessarily maximize the Touschek lifetime. Shown in Figure $2 b$ are the potential wells and distributions for the conditions in Figure 2a except with a \pm 0.5 degree phase shift, indicating the sensitivity of the flattened potential well to small variations. If the voltage is increased beyond the flattened potential well condition, two stable fixed points are formed and the bunch forms two bunchlets within the RF bucket.

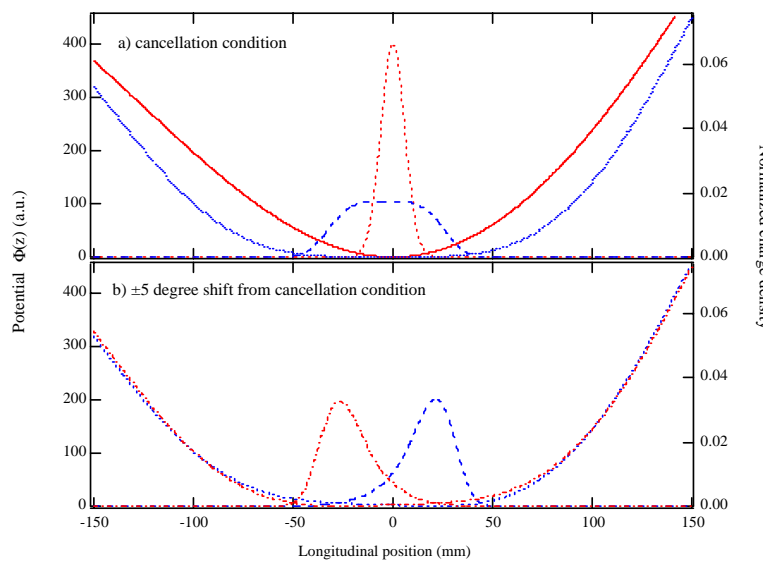

Figure 2. Potential well and longitudinal distribution for a) nominal RF voltage and optimized harmonic voltage and b) optimized voltage with small phase variation.

Short-range wake voltages generated by the broadband impedance of the ring also affect the bunch lengthening, particularly near optimum flattening of the potential well when the bunch shape is most sensitive to small perturbations. However, as discussed below, the ALS harmonic cavities cannot be operated near optimum bunch lengthening for most of a fill. Therefore, we neglect these effects in this paper.

If the harmonic voltage shown in Fig. 1 were phased such that the slopes of the main and harmonic voltages added rather than cancelled, the bunch would be shortened.

\subsection{Passive cavity operation}

A particularly attractive option for improving the lifetime is to lengthen the bunches using a passive harmonic cavity. The voltage in a passive cavity is generated by the beam itself and thus an external RF source is not required. Because the harmonic cavities do not accelerate the beam, there are interesting arguments for the use of either normal or superconducting cavities.[3] However, the main difficulty of a passive harmonic system is that the effect can vary greatly with total beam current.

The tuning of the cavity for the case for bunch lengthening is illustrated in Figure 3. The cavity is tuned above the Fourier component of the beam current to induce a phase shift between the beam and voltage of almost 90 degrees. For bunch shortening, the cavity is tuned below the beam component. Thus the amplitude and phase of the harmonic voltage are adjusted by tuning the resonant frequency of the passive harmonic cavity. However, for a fixed cavity Q and shunt impedance, the amplitude and phase cannot be independently adjusted. Thus the optimum lengthening conditions can be achieved at only a single beam current, if at all. This is one of the primary limitations of passive operation.

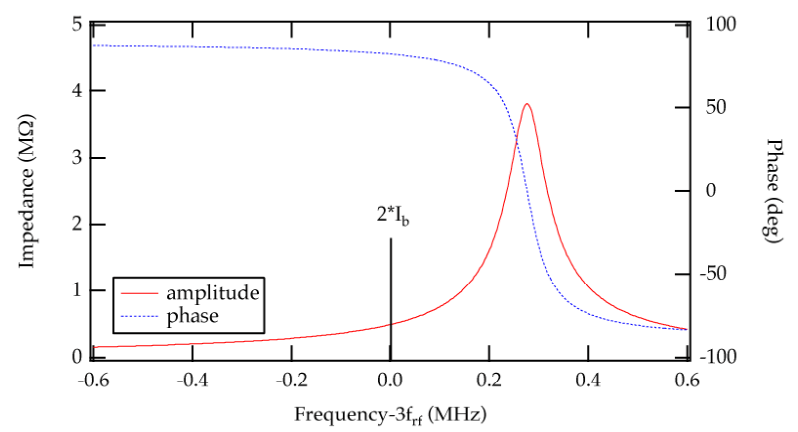

Figure 3. Tuning of harmonic cavity fundamental with respect to Fourier harmonic of beam current.

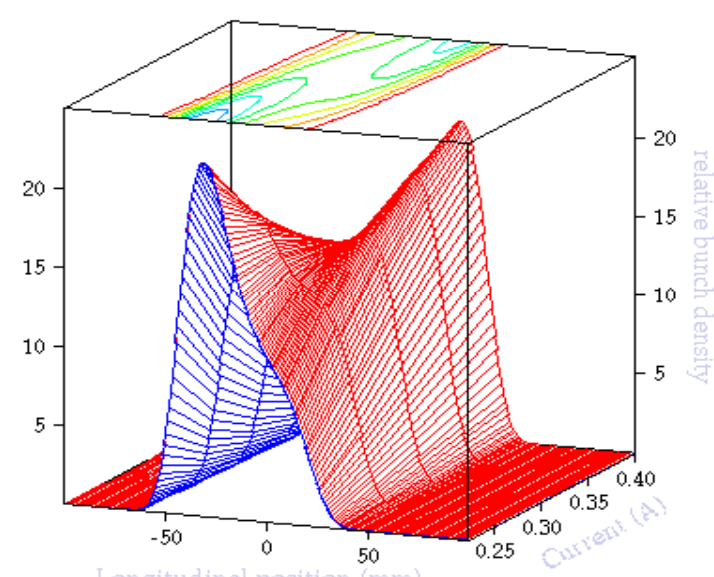

Figure 4. Bunch shape over the for a fixed harmonic voltage from 0.4-0.2 A. The bunch shape and phase change significantly vs. current.

For example, for ALS conditions at $1.9 \mathrm{GeV}$ we estimate a requirement of $3.8 \mathrm{M} \Omega$ harmonic impedance at 0.4 A to reach optimum flattening, resulting in a factor 3.1 lifetime improvement. Our harmonic cavity design has an $\mathrm{R} / \mathrm{Q}=80.4 \Omega$ and a measured $\mathrm{Q}$ of $21000[1]$. Using this cavity, we calculate that we can achieve optimum flattening using 3 cells at $320 \mathrm{~mA}$.

One of the potential disadvantages of the passive cavity is that the bunch shape and synchronous phase are not kept constant over a fill. This is demonstrated in Fig. 4 which shows the evolution of bunch shape and position relative to the main RF phase over a fill with the cavites for a fixed harmonic voltage. For the more optimal case of a fixed voltage, when the beam current is not near the optimum condition, the bunch position slips away from 
the nominal synchronous phase. This may be a nuisance to some synchrotron radiation experiments which use the time structure of the bunch in the experiments. Fortunately, the phase slip occurs slowly over the course of a fill. There is much less change in the bunch shape and phase over a fill for the case of the fixed detuning. The conclusion is that when operating passive harmonic cavities closer to maximum bunch lengthening, the bunch shape and phase are much more sensitive to the beam current and can vary significantly.
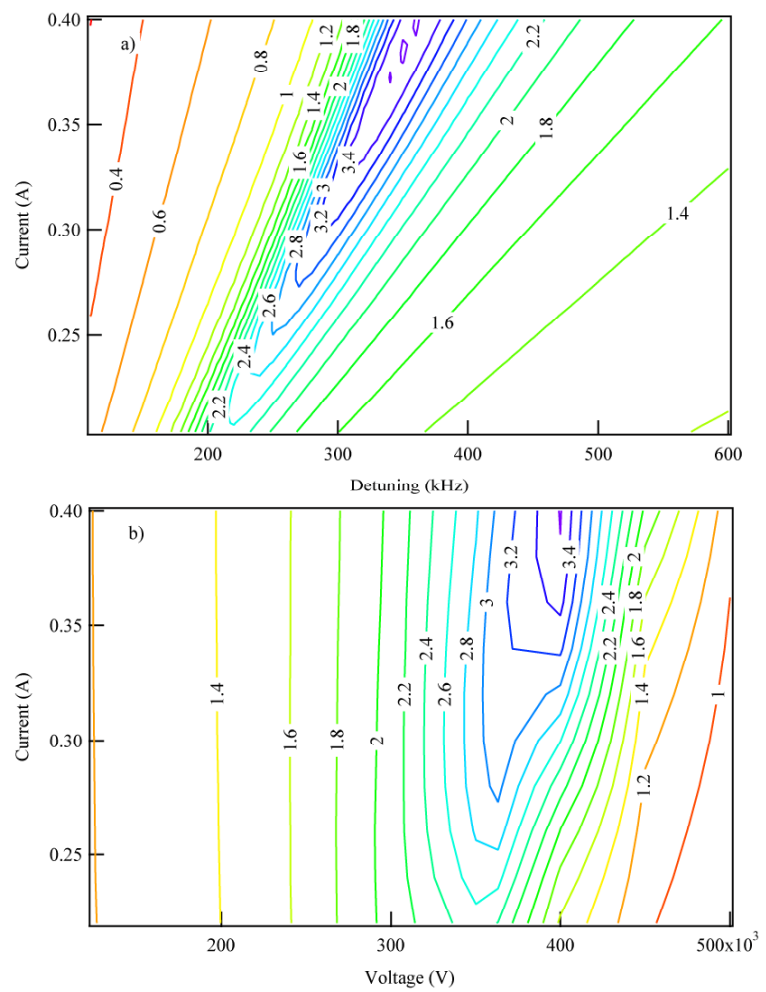

Figure 5. Contour plots of lifetime improvement vs. a) detuning and $b$ )fixed voltage.

To optimize the lifetime performance over a fill, we can consider the two simplest operating scenario. The first is to keep the cavities detuned a fixed amount as a function of beam current and the second is to detune the cavities as to maintain a constant cell voltage. Figure 5 shows the lifetime improvement as a function of a) harmonic detuning and b) constant harmonic voltage.

One can quantify the overall effect in terms of the ratio of the integrated current with to without harmonic cavities[3]. The fixed voltage realizes about a $10 \%$ improvement over a fixed detuning. However, it may be more practical to operate with a fixed detuning for practical reasons such as mechanical limitations of tuners and tuning of cavity higher order modes.

\section{ALS OPERATING EXPERIENCE}

Our primary diagnostics for measuring the effects of the harmonic cavities were a streak camera (SC), a DCCT current monitor from which the lifetime could be measured, and a spectrum analyzer for examining beam signals. The SC is a Hamamatsu dual-axis synchroscan streak camera which allows measurement of the bunch length and relative synchronous phase for all of the bunches in the bunch trains used in our experiments. All of the measurements shown in this paper were made at $1.9 \mathrm{GeV}$ using fill patterns with all buckets filled except for either a $2.51 \%$ or $171 \%$ gap. Furthermore, in all of the results shown below the beam is longitudinally and transversely stable except where indicated. This is important to note because the lifetime in the ALS depends strongly on the effective beam dimensions and thus the effect of the harmonic cavities is best understood when the beam is stable.

To date, we have measured lifetime improvements of over a factor two in ideal conditions during machine studies and about 50\% during user operation with corresponding amounts of bunch lengthening

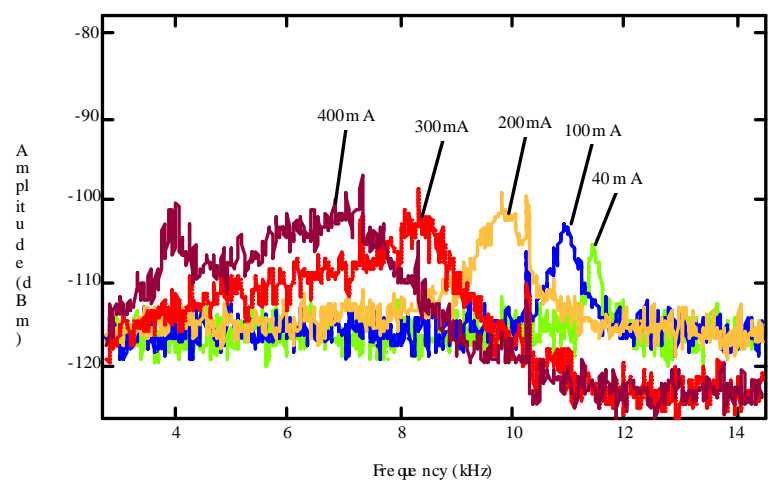

Figure 6. Synchrotron mostion observed on a spectrum analyzer for fixed detuning vs. current. The synchrotron frequency decreases by almost a factor two and broadens considerably.

We also observed a large detuning and of the synchrotron frequency as we varied the voltage in the cavities. Shown in Fig. 6 is the amplitude of a longitudinal beam transfer function made with 3 cavities in lengthening mode and 2 parked, measured in the small gap filling pattern. In this case, the cavities were left detuned at $+0.5^{*} \mathrm{f}_{0}$ and only the beam current was varied. At low current the synchrotron frequency is at the nominal value. As the current increases, the frequency decreases and the width of the response increases. We believe the increase in width to correspond to the increase in spread of synchrotron frequencies within the bunch. The limit to decreasing the synchrotron frequency occurs when the width of the beam response falls outside the bandwidth of the digital filter used in the longitudinal feedback (LFB) system.

We observed a large variation in the relative phase of each bunch when the harmonic cavities were tuned with their nominal voltages and with a large gap in the beam fill pattern. A measurement of the relative beam phase extracted from a SC image is shown in Fig. 7a. Note that there is also a variation in the bunch length along the bunch train as shown in Fig. 7b. Both of these effects are reduced for a small gap in the fill pattern. This beam phase transient and variation in bunch length has been the 
one of the limiting factors in achieving the theoretical maximum lifetime increase. This is discussed in detail in the next section.

We also observe some Landau damping of the longitudinal instabilities created by either an interbunch or intrabunch spread in synchrotron frequencies. Early in the commissioning process we had difficulty operating the harmonic cavities together with the LFB and began operating them with the LFB turned off and the beam unstable. When the beam current was above about 320 $\mathrm{mA}$, we observed a sudden collapse in the beam size, indicating a sharp decrease in the amplitude of unstable oscillations. We discovered that this effect would only occur for beam fill patterns with gaps. This probably means that the Landau damping is coming from a spread in synchrotron tunes between bunches and not within a bunch.

This effect also results in an interesting hysteretic phenomenon. When the beam is longitudinally unstable, the beam current harmonic driving the cavities is reduced due to the phase modualtion of the beam and results in a lower voltage. However, once the voltage reaches a certain level and the beam is stabilized via Landau damping, the beam harmonic grows, the voltage increases, and the beam is subsequently stable down to a current much below where it initially stabilized. We have not found this effect to be useful in the ALS because of the widely varying beam current at which the beam stabilizes and its strong dependency on the fill pattern.

The LFB is also affected by the downward shift and increasing width of the synchrotron resonance. To achieve correct feedback phase and gain, the digitized bunch phase information is processed through a digital filter implemented as an array of general-purpose digital signal processors. The original control filter in use before the addition of the third harmonic cavities was a finite impulse response (FIR) filter centered at the nominal synchrotron frequency with the phase adjusted to optimize the damping. The filter in Fig. 6a has usable control bandwidth of $2.7 \mathrm{kHz}$. To accommodate a larger range of synchrotron frequencies we designed an infinite impulse response (IIR) filter with a $6.5 \mathrm{kHz}$ control bandwidth. Its frequency response is shown in Fig. $6 \mathrm{~b}$.

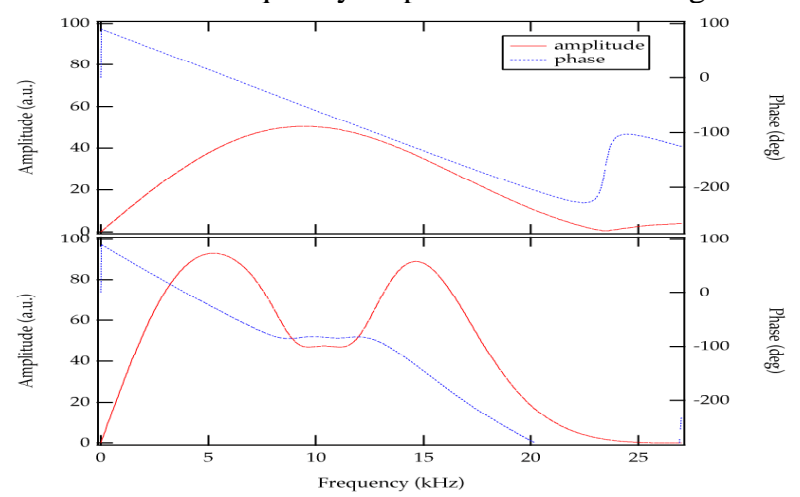

Figure 7. Frequency response of digital filters used in the ALS multibunch longitudinal feedback. Top)
Narrowband filter. Bottom) Broader band filter which allows wider range of synchrotron frequency.

\section{BEAM LOADING TRANSIENTS}

As described in the previous section, the beam loading transients observed with the harmonic cavities were much larger than expected and presented a number of technical challenges and, in our opinion, a severe limitation to achieving maximum bunch lengthening and lifetime increase. Common beam loading models are not adequate to describe the effects with harmonic cavities[4]. For this reason, we have developed a simple computer tracking code in which we compute the transient voltages in the main and harmonic RF systems and use this information to compute the shape of each bunch and thus the mean lifetime improvement. Using this code we can model the effects of normal and superconducting cavities, variations in beam fill patterns, Robinson instabilities, and the Landau damping of interbunch tune spreads. However, one of the limitations of the code is that it models each bunch as a macroparticle and thus neglects intrabunch tune spreads. This section presents a qualitative description of the transient behavior and a summary of our results.

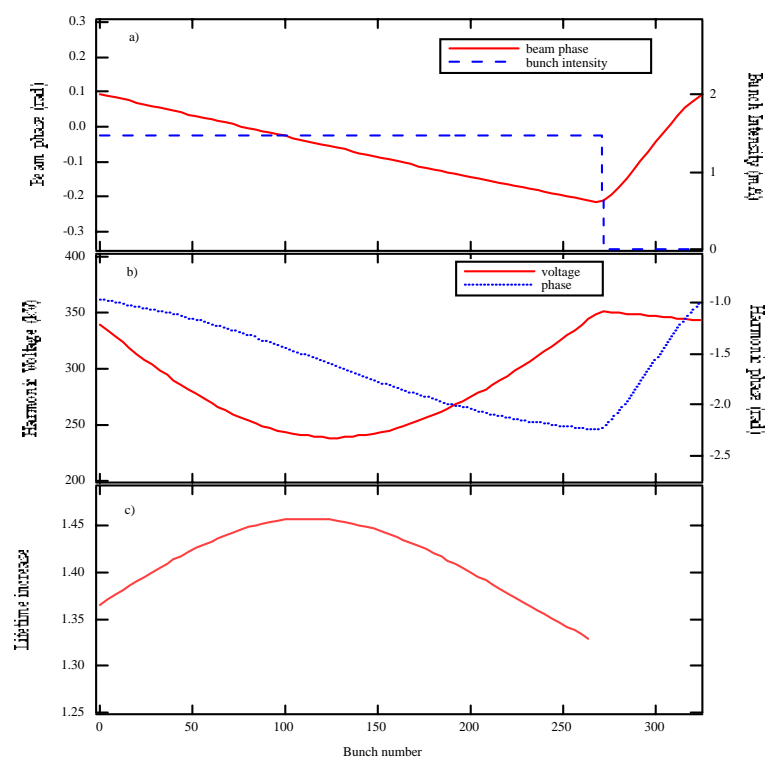

Figure 8. Simulation results for ALS conditions with 17\% gap in the fill pattern.

Figure 8 shows a result for the ALS conditions with the harmonic cavities tuned to give a factor 2 in lifetime improvement (with no phase transient) and a 17\% gap in the fill pattern. Fig. 8a shows the steady-state distribution of the synchronous phase along the bunch train with a total phase transient of about $0.3 \mathrm{rad}$, resulting from the transient beam loading of the main and the harmonic cavity. Fig. $8 \mathrm{~b}$ shows the strong modulation of the harmonic voltage and phase which reaches $\pm 17 \%$ in amplitude and $\pm 0.6 \mathrm{rad}$. As a consequence, the amplitude and phase relations between the main and the harmonic cavity that are required to reduce the slope of the total RF 
voltage are more or less altered along the bunch train. This leads to a variation in the bunch lengthening along the train, as depicted in Fig. 8c. The maximum appears in the middle of the bunch train because that is where the phase is such that the bunch is lengthened. Even the maximum bunch lengthening of about 1.5 for the fill with gap is lower than the value of 2.12 obtained under similar operation conditions for uniform filling. In this case, the average bunch lengthening is less than 1.4 and bunches at the edge of the fill experience a stretching of only $30 \%$. These results agree well with observations made at the ALS and at BESSY and indicate that the transient loading can cause degradation of the lifetime improvement.

The transient loading in the harmonic cavities has some comparison with that in the main RF system. The total phase transient scales with the total R/Q of the system, the beam current, and the size of the gap. Note that the transients do not scale with the $Q$ and thus superconducting (SC) cavities will also suffer from these effects. However, the transients do not scale similarly with detuning because in the case of the harmonic cavities, the induced voltage significantly distorts the response of the beam, shifting the synchrotron frequency by up to a factor three. This is the main reason the small-signal modulation (Pedersen) model is not accurate for harmonic cavities. For example, note the time dependence of the voltage transient is much different than that in the fundamental. In the harmonic cavity, the voltage initially decreases and then increases. This is because the beam phase shift is so large along the bunch train that the bunch head and tail not excite voltage out of phase with each other.
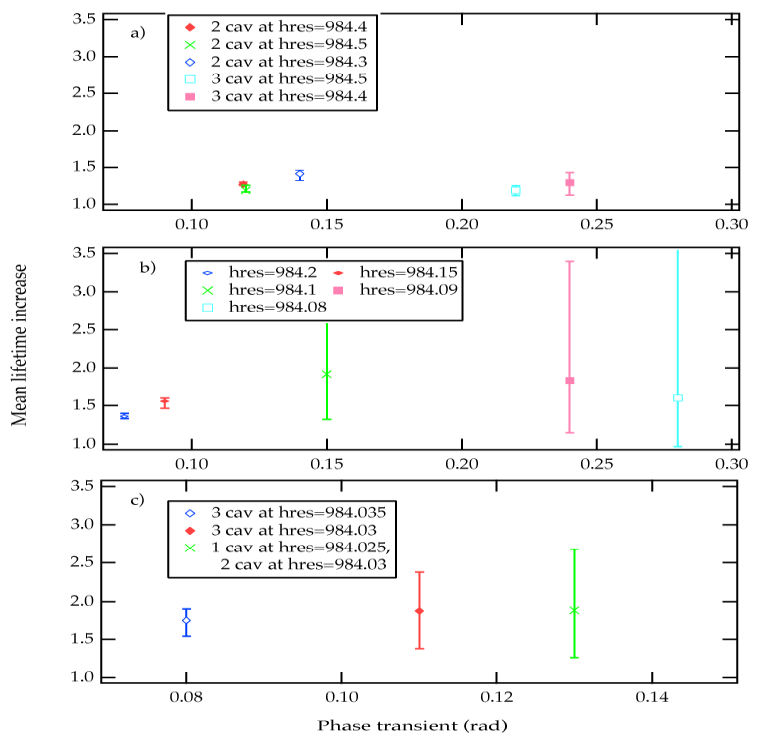

Figure 9. Lifetime improvement vs. total phase transient. The error bars indicate the range of increase along the bunch train. a) Two and three NC cavities. b) Three

ARES-type structures and c) One SC cavity.

A summary of our results for passive cavities is shown in Fig. 9. In addition to normal and super-conducting cavities, we also investigated ARES-type storage cavities.
The conclusion is that passive storage cavities and SC cavities give that best results for ALS conditions but still well below the optimum results.

\section{SUMMARY AND ACKNOWLEDGEMENTS}

I would like to thank numerous people for contributing during various phases of the harmonic cavity project. These Bob Rimmer, Mark Franks, David Plate, C. C. Lo, Ken Baptiste, Jim Julian, and the ALS accelerator physics and operations groups. I would also like to thank Nathan Towne for many useful discussions.

\section{REFERENCES}

[1] J. M. Byrd, K. Baptiste, S. De Santis, S. Kosta, C. C. Lo, D. Plate, R. A. Rimmer, M. Franks, Nucl. Inst. Meth. A (439) 1(1999) pp. 15-25.

[2] J. M. Byrd, S. De Santis, M. Georgsson, G. Stover, J. D. Fox, D. Teytelman, Nucl. Inst. Meth. A, (455) 2(2000) pp. 273284.

[3] J. M. Byrd, M. Georgsson, Phys. Rev. ST Accel. Beams 4 030701 (2001).

[4] J. M. Byrd, S. De Santis, J. Jacob, V. Serriere, submitted to Phys. Rev. ST Accel Beams.

[5]R. Biscardi, S. L. Kramer, G. Ramirez, Nucl. Inst. Meth. A 26026 (1995). S. Krinsky, et. al., AIP Conf. Proc. 249, 840 (1990). J. Keane, et. al., Proc. of the 1989 Part. Acc. Conf., Vancouver, B.C., 1989.

[6]K. Kleman, Proc. of the 1995 Part. Acc. Conf., Dallas, 1795 (1995).

[7] ̊.. Andersson, MAX preprint (NTMX-7009) (1988). M. Georgsson, Å. Andersson, M. Eriksson, Nucl. Inst. Meth. A 416 465 (1998).

[8]C. Wang, et. al., Proc. of the 1997 Part. Acc. Conf., Vancouver, B.C., 1997.

[9]M. Georgsson, , W. Anders, D. Krämer, J. M. Byrd, "Design and commissioning of 3rd harmonic cavities at BESSY II" to appear in , Nucl. Inst. Meth.. A.

[10] M. Svandrlik, et. al., Proceedings of 2000 European Particle Accelerator Conference, EPAC2000, Berlin, p. 2052 (2000).

[11] S. Bartalucci, M. Migliorati, L. Palumbo, B. Spartaro and M. Zobov, Proc. of the European Particle Accelerator Conference, London, England, 1994.

[12] G. Flynn, et. al., Proc. of the 1998 Euro. Part. Acc. Conf., Stockholm, 954 (1998).

[13] R. A. Bosch, C. S.Hsue, Particle Accelerators 42, 81 (1993).

[14]M. Migliorati, L. Palumbo, M. Zobov, Nucl. Inst. Meth. A (354) 1(1995) pp. 215-223.

[15]A. Hofmann and S. Myers, Proc. of the 11th Int. Conf. on High Energy Acc. ISR-TH-RF/80-26 (1980).

[16] T.-S. Wang, Proc. of the 15th international Conference on High Energy Accelerators, Hamburg, Germany, 1992. T.-S. Wang Los Alamos preprint AT-7, ATN-92-11, (1992). 